\title{
AUTO-AVALIAÇÃO DA AUDIÇÃO EM IDOSOS
}

\author{
Self-evaluation of hearing in elderly
}

\author{
Lívia Maria Santiago ${ }^{(1)}$, Cristiane de Oliveira Novaes ${ }^{(2)}$
}

\section{RESUMO}

Objetivo: investigar queixas auditivas e caracterizar impactos sociais e emocionais do handicap auditivo em idosos. Métodos: participaram deste estudo transversal, 35 idosos dos projetos Enriquecimento Cultural e Nucleação, ambos do Pólo Interdisciplinar do Envelhecimento da Universidade Federal de Juiz de Fora. Utilizou-se dois instrumentos de coleta de dados: o questionário sócio-demográfico, de saúde geral e percepção auditiva e o de avaliação da desvantagem auditiva The Hearing Handicap Inventory for the Elderly, HHIE (adaptado por Wieselberg, 1997). Efetuou-se a análise descritiva das variáveis de estudo e, para verificar a associação entre a ocorrência de desvantagem auditiva com as demais variáveis, realizou-se análises bivariadas, por meio do teste $X^{2}$, considerando $p \leq 0.05$. Resultados: foram observadas queixas auditivas para $11(31,4 \%)$ indivíduos e de desvantagem auditiva (social e/ou emocional) para $10(28,6 \%)$. As principais implicações da desvantagem auditiva relacionaram-se às habilidades sociais, compreensão da fala durante atos comunicativos; dificuldades na compreensão da TV e/ou rádio; baixa tolerância a sons intensos e a ambientes ruidosos. Apenas sete $(20 \%)$ entrevistados não apresentaram nenhuma percepção de desvantagem. Conclusão: apesar de ter sido utilizada amostra reduzida, foram observadas queixas auditivas em atividades cotidianas dos idosos, que dificilmente seriam pesquisadas em avaliações de saúde de rotina, nem mesmo em audiometrias. Os resultados foram expressivos, tendo em vista a utilização de amostra específica de idosos e o predomínio de idosos "mais jovens", fatos que podem subestimar as queixas.

DESCRITORES: Auto-avaliação; Audição; Envelhecimento

\section{INTRODUÇÃO}

De todos os aspectos relacionados ao envelhecimento populacional, a saúde ainda é o principal alvo de estudos e intervenções. As razões dessa preocupação são as especificidades do envelhecimento, como a alta prevalência de problemas crônicos e/ou degenerativos observada em diversos estudos ${ }^{1,2}$.

O declínio auditivo é considerado uma condição incapacitante, que limita ou impede o indivíduo de desempenhar seu papel social de forma plena e integrada e a audição é apontada como o primeiro dos sentidos a apresentar perdas funcionais detectáveis de maneira objetiva, sendo que, em média, é

(1) Fonoaudióloga; Especialista em Políticas e Pesquisa em Saúde Coletiva; Mestranda do Programa de Saúde Pública e Meio Ambiente da Escola Nacional de Saúde Pública Sérgio Arouca, Fiocruz.

(2) Psicóloga; Mestre em Ciências da Saúde; Doutoranda do Programa de Saúde Pública e Meio Ambiente da Escola Nacional de Saúde Pública Sérgio Arouca, Fiocruz. por volta dos 30 anos que se inicia o processo de envelhecimento auditivo ${ }^{3}$. Mesmo que tal fenômeno ocorra de maneira lenta, o impacto na vida do indivíduo idoso é considerável, visto que a deficiência auditiva, somada as mais diversas mudanças (biológicas, psicológicas e sociais), pode levar a uma queda na qualidade de vida e até ao isolamento social, ocasionado pelo declínio da capacidade de comunicação ${ }^{3-5}$.

Cabe dizer que a diminuição da audição é condição frequente na população idosa. Um estudo brasileiro aponta para uma prevalência de queixa de dificuldade auditiva entre os idosos de $31,8 \%{ }^{6}$. Durante o processo de envelhecimento, associados à diminuição da audição, há outros problemas como a incapacidade auditiva, que é a inabilidade de perceber a fala em ambientes ruidosos e a desvantagem auditiva, referindo-se aos aspectos emocionais e sociais que impedem o indivíduo de desempenhar adequado papel comunicativo na sociedade ${ }^{7}$.

Cada vez mais são utilizados: entrevistas, questionários ou questões isoladas de auto-avaliação da audição, para que seja possível quantificar as 
dimensões subjetivas e qualitativas da perda auditiva e/ou realizar triagem de pacientes com queixas, para posterior realização da avaliação audiométrica, caso esta seja necessária ${ }^{5,8}$.

Este estudo buscou investigar queixas auditivas e caracterizar impactos sociais e emocionais do handicap auditivo em idosos em uma amostra de idosos de Juiz de Fora, MG.

\section{MÉTODOS}

Foram entrevistados indivíduos com idade igual ou superior a 60 anos, participantes de dois projetos do Pólo Interdisciplinar na Área do Envelhecimento (PIE) da Universidade Federal de Juiz de Fora (UFJF). Os projetos são:

- Enriquecimento Cultural e Processo de Envelhecimento, destinado a pessoas alfabetizadas com idade a partir de 45 anos do município e região, é desenvolvido na sede do Pólo Interdisciplinar na Área do Envelhecimento (PIE), objetivando fornecer aos seus participantes, informações para compreensão e acompanhamento do debate da sociedade moderna, contextualizando-os no mundo contemporâneo, preparando-os para assumirem o seu processo de envelhecimento, visando o fortalecimento de sua identidade $e$ cidadania, a melhoria da qualidade de vida e de participação social na comunidade local;

- Nucleação, realizado pelo PIE em bairros específicos do município de Juiz de Fora, com o apoio da SESU/MEC - PROEXT/2003, destinado a idosos de comunidades carentes dos bairros da zona oeste de Juiz de Fora, objetivando verificar as demandas da população idosa do bairro Dom Bosco e Martelos e a adesão da mesma a atividades educativas, preventivas e culturais realizadas no próprio bairro. O projeto foi estruturado a partir do levantamento da demanda e estabelecimento de parceria nos bairros e a realização de atividades quinzenais (palestras educativas, oficinas, atividades sócio-culturais e ações assistenciais).

Durante um encontro de cada um dos projetos, os idosos participantes foram abordados com a apresentação da proposta da pesquisa, esclarecimentos a respeito dos procedimentos envolvidos e convite à participação. Aqueles que aceitaram o convite foram entrevistados no próprio local no final do encontro. A participação no estudo foi formalizada por meio da assinatura do Termo de Consentimento Livre e Esclarecido (TCLE).
Os critérios de inclusão da população de estudo foram: ter idade igual ou superior a 60 anos; assinar o TCLE pessoalmente ou por meio de representante; declarar estar apto e interessado em participar da pesquisa; participar de um dos dois projetos do PIE pré-selecionados.

Foram utilizados dois instrumentos de coleta de dados. O primeiro constou de uma entrevista estruturada, que abordou variáveis distribuídas em três categorias: variáveis sócio-demográficas (idade, sexo, estado civil, escolaridade, situação profissional, moradia e renda média familiar); variáveis relacionadas às condições de saúde gerais (saúde auto-referida geral e referenciada a visão, audição e memória, tipo de serviço de saúde utilizado, utilização de medicação, comportamentos danosos à saúde, avaliação de funções e queixas auditivas).

O segundo instrumento utilizado foi o questionário de auto-avaliação do Handicap Auditivo para Idosos (The Hearing Handicap Inventory for the Elderly- HHIE, Ventry \& Weinstein, 1982- Versão adaptada por Wieselberg, 1997), que se trata de um instrumento que objetiva avaliar a auto-percepção da desvantagem auditiva nos idosos, composto de 25 questões abordando domínios das habilidades sociais e emocionais, relativas às dificuldades auditivas provenientes do envelhecimento, contando com uma escala de resposta que varia de 0 a $100^{\circ}$. A apuração dos resultados foi realizada por meio da atribuição de pontos que varia de 0 a 4 pontos para cada questão, sendo que a resposta sim equivale a 4; e não equivale a zero. Sendo que o escore geral é compreendido entre zero (ausência de desvantagem) e 100 pontos (máxima percepção de desvantagem auditiva). Os valores compreendidos entre 0 e 16 pontos significam que não há percepção de desvantagem auditiva. Entre 18 e 42 pontos, há percepção leve/moderada de desvantagem auditiva e entre 42 e 100 pontos há percepção severa/significativa de desvantagem auditiva ${ }^{9}$.

O estudo foi aprovado pelo Comitê de Ética em Pesquisa da Universidade Federal de Juiz de Fora, sob número de protocolo $268 / 2005$, de acordo com as diretrizes para a pesquisa envolvendo seres humanos contidas na Resolução CNS, nº 196/96. Foram efetuadas as análises descritivas das variáveis de estudo, por meio de frequência simples. Para verificar a associação entre a ocorrência de desvantagem auditiva com as demais variáveis, realizou-se análises bivariadas, por meio do teste $\mathrm{X}^{2}$, considerando $\mathrm{p} \leq 0.05$.

As análises foram realizadas utilizando-se o pacote estatístico SPSS 10.0 (Statistical Package Social Science). 


\section{RESULTADOS}

Foram entrevistados 35 idosos, com idades entre 60 e 88 anos, dos quais 32 eram do sexo feminino. Em sua maioria, eram participantes do Projeto Nucleação do Pólo Interdisciplinar na Área do Envelhecimento (PIE), nos bairros Dom Bosco e Martelos, Juiz de Fora, MG.

Em relação ao estado civil, os casados correspondiam a $18(48,6 \%)$ da amostra e os viúvos a 13 (40\%). Quanto à escolaridade, $16(45,7 \%)$ afirmavam ter o ensino básico concluído. Nenhum dos entrevistados trabalhava, mas $16(74,3 \%)$ tinham renda própria. O valor médio da renda familiar de 17 $(54,5 \%)$ dos participantes encontrava-se entre um e dois salários mínimos e para oito $(24,2 \%)$ entre três e quatro salários (Tabela 1).

Na auto-avaliação da saúde predominou a categoria razoável $15(42,9 \%)$ e boa $13(37,1 \%)$ na amostra. A respeito da visão, $17(48,6 \%)$ da amostra a avaliaram como razoável, sendo que $25(71,4 \%)$ usavam óculos. A auto-avaliação da audição resultou em boa para $10(28,6 \%)$ e razoável para oito $(22,9 \%)$ dos idosos, sendo que apenas uma $(2,9 \%)$ participante da pesquisa usava prótese auditiva. A memória foi avaliada como boa para $16(45,7 \%)$ dos sujeitos entrevistados, e 24 (68,6\%) afirmavam não necessitar de ajuda de outras pessoas para desempenhar suas atividades diárias (Tabela 2).

Grande parte da população declarou alguma morbidade e referiu o uso regular de medicamentos, predominando sujeitos que utilizavam até dois medicamentos. A indicação mais frequente era para hipertensão arterial. As condições crônicas mais expressivamente relatadas eram as doenças

Tabela 1 - Características sócio-demográficas, 35 idosos participantes de projetos da UFJF

\begin{tabular}{|c|c|c|c|}
\hline Variável & Categorias & $\mathrm{N}$ & $\%$ \\
\hline \multirow[t]{3}{*}{ Local da entrevista } & Bairro Dom Bosco & 17 & 48,6 \\
\hline & PIE & 12 & 34,3 \\
\hline & Bairro Martelos & 6 & 17,1 \\
\hline \multirow[t]{2}{*}{ Sexo } & Masculino & 3 & 8,6 \\
\hline & Feminino & 32 & 91,4 \\
\hline \multirow[t]{3}{*}{ Estado civil } & Solteiro/divorciado & 4 & 11,4 \\
\hline & Casado & 18 & 48,6 \\
\hline & Viúvo & 13 & 40,0 \\
\hline \multirow[t]{2}{*}{ Escolaridade } & 8 anos ou menos & 29 & 82,9 \\
\hline & Mais de 8 anos & 6 & 17,1 \\
\hline \multirow[t]{2}{*}{ Trabalha } & Sim & 0 & 0,0 \\
\hline & Não & 35 & 100,00 \\
\hline \multirow[t]{2}{*}{ Renda própria } & Sim & 26 & 74,3 \\
\hline & Não & 9 & 25,7 \\
\hline \multirow[t]{3}{*}{ Fonte de renda } & Aposentadoria & 27 & 82,9 \\
\hline & Pensão & 7 & 11,4 \\
\hline & Ajuda de parentes & 3 & 5,7 \\
\hline \multirow[t]{4}{*}{ Total de renda } & 1 a 2 salários & 17 & 54,5 \\
\hline & 3 a 4 salários & 8 & 24,2 \\
\hline & 5 a 8 salários & 1 & 3,0 \\
\hline & mais de 8 salários & 6 & 18,2 \\
\hline \multirow[t]{3}{*}{ Tipo de moradia } & Alugada & 5 & 14,3 \\
\hline & Cedida & 6 & 17,1 \\
\hline & Própria & 24 & 68,6 \\
\hline \multirow[t]{4}{*}{ Mora com outros? } & Sozinho & 7 & 20,0 \\
\hline & Com 1 pessoa & 6 & 17,1 \\
\hline & Com 2 pessoas & 11 & 31,4 \\
\hline & Com 3 ou mais pessoas & 11 & 31,4 \\
\hline
\end{tabular}

* As diferenças nos valores absolutos correspondem aos valores missing 
Tabela 2 - Características relativas ao status de saúde, 35 idosos participantes de projetos da UFJF

\begin{tabular}{|c|c|c|c|c|}
\hline \multicolumn{2}{|c|}{ Grupo de variáveis } & \multirow{2}{*}{$\begin{array}{c}\text { Categorias } \\
\text { Muito Boa }\end{array}$} & \multirow{2}{*}{$\begin{array}{l}N \\
6\end{array}$} & \multirow{2}{*}{$\begin{array}{c}\% \\
17,1\end{array}$} \\
\hline Auto-avaliação & Saúde & & & \\
\hline & & Boa & 13 & 37,1 \\
\hline & & Razoável & 15 & 42,9 \\
\hline & & Ruim & 1 & 2,9 \\
\hline & Visão & Muito Boa & 2 & 5,7 \\
\hline & & Boa & 13 & 37,1 \\
\hline & & Razoável & 17 & 48,6 \\
\hline & & Ruim & 3 & 8,6 \\
\hline & Audição & Excelente & 7 & 20,0 \\
\hline & & Muito Boa & 7 & 20,0 \\
\hline & & Boa & 10 & 28,6 \\
\hline & & Razoável & 8 & 22,9 \\
\hline & & Ruim & 3 & 8,6 \\
\hline & Memória & Excelente & 3 & 8,6 \\
\hline & & Muito Boa & 5 & 14,3 \\
\hline & & Boa & 16 & 45,7 \\
\hline & & Razoável & 9 & 25,7 \\
\hline & & Ruim & 2 & 5,7 \\
\hline \multirow{2}{*}{\multicolumn{2}{|c|}{ Refere algum problema de saúde }} & $\operatorname{Sim}$ & 27 & 77,1 \\
\hline & & Não & 8 & 22,9 \\
\hline \multirow{2}{*}{\multicolumn{2}{|c|}{ Medicação }} & Sim & 33 & 94,3 \\
\hline & & Não & 2 & 5,7 \\
\hline \multirow{2}{*}{\multicolumn{2}{|c|}{ Tabagismo atual }} & Sim & 2 & 5,7 \\
\hline & & Não & 33 & 94,3 \\
\hline \multirow{2}{*}{\multicolumn{2}{|c|}{ Consumo de álcool }} & Sim & 2 & 5,7 \\
\hline & & Não & 33 & 94,7 \\
\hline
\end{tabular}

* As diferenças nos valores absolutos correspondem aos valores missing

cardiovasculares, seguidas das ósteo-articulares e das metabólicas. Em situações de necessidade de atendimento de saúde, o tipo de serviço predominantemente utilizado era o público, representando $25(71,4 \%)$ do total. Quanto aos hábitos de risco, $33(94,3 \%)$ dos idosos não eram tabagistas e 33 $(94,3 \%)$ relataram não consumirem bebidas alcoólicas (Tabela 2).

Quando comparados os grupos de indivíduos por projeto ao qual participavam, observa-se que o nível de escolaridade e a média de renda familiar dos idosos do projeto Nucleação eram inferiores aos dos idosos do Projeto Enriquecimento Cultural e Processos do Envelhecimento.

As principais queixas auditivas e sua distribuição estão descritas na Tabela 3. Nos dados obtidos por meio da análise do questionário HHIE foi observado que $25(71,4 \%)$ de pesquisados não tinham percepção de desvantagem auditiva, oito $(22,9 \%)$ tinham percepção leve/moderado e dois $(5,7 \%)$ com percepção severa/significativa. Sendo que somente sete (20\%) indivíduos da amostra apresentou pontuação zero, ou seja, ausência total de percepção da desvantagem.

Para 28 (80\%) dos entrevistados, todas as questões relativas aos aspectos sociais apresentaram respostas negativas, excetuando-se a questão S-8 (Você sente dificuldade em ouvir quando alguém fala cochichando?) que obteve $13(37,1 \%)$ de resposta afirmativa e $10(28,6 \%)$ de resposta "às vezes". Quanto às questões relativas aos aspectos emocionais, foram observadas respostas afirmativas para percepção de desvantagem auditiva entre $10(28,6 \%)$ e dois $(5,7 \%)$ para todas as questões.

Em relação às queixas auditivas por faixas etárias pode-se observar que os idosos de 70 a 79 anos e os acima de 80 anos, quando comparados aos indivíduos de 60 a 69 anos, apresentaram duas queixas significativas: sentem mais necessidade de que as pessoas repitam o que disseram $(p=0,011)$ e de colocar a TV e o rádio em volumes mais altos para ouvirem melhor $(p=0,044)$. Ao se analisar a 
Tabela 3 - Queixas auditivas, 35 idosos participantes de projetos da UFJF

\begin{tabular}{lccc}
\hline Variável (questão) & Categorias & N & $\%$ \\
\hline Ouve o que as pessoas dizem a você? & Sim & 30 & 91,2 \\
Compreende tudo o que as pessoas dizem a você? & Não & 5 & 8,81 \\
& Sim & 22 & 62,9 \\
Necessita que as pessoas repitam o que disseram a & Não & 13 & 37,1 \\
você? & Sim & 14 & 42,9 \\
Ouve os sons das buzinas dos carros, do apito dos & Não & 19 & 57,1 \\
guardas, de sirenes, etc? & Não & 32 & 94,3 \\
Ouve e compreende conversas ao telefone? & Sim & 3 & 5,7 \\
& Não & 30 & 88,2 \\
Escuta e compreende os sons da TV? & Sim & 4 & 11,8 \\
& Não & 30 & 85,3 \\
Escuta e compreende os sons do rádio? & Sim & 30 & 14,7 \\
& Não & 3 & 85,3 \\
Tem necessidade de colocar a TV e o rádio em & Sim & 11 & 14,7 \\
volumes altos, para ouvir melhor? & Não & 23 & 31,4 \\
Sente irritação, desconforto ou dor nos ouvidos em & Sim & 11 & 68,6 \\
ambientes barulhentos? & Não & 24 & 34,3 \\
Sente irritação, desconforto ou dor nos ouvidos ao & Sim & 17 & 65,7 \\
ouvir sons muito altos? & Não & 18 & 48,6 \\
\hline
\end{tabular}

* As diferenças nos valores absolutos correspondem aos valores missing

desvantagem auditiva por faixas etárias, pode-se observar que os idosos de 70 a 79 anos e a acima de 80 anos referem assistir à TV ou ouvir o rádio menos do que gostariam $(p=0,013)$.

Ao comparar os idosos de ambos os projetos (Projeto Enriquecimento Cultural e Processos do Envelhecimento e Nucleação), foram encontradas diferenças significativas quanto à questão a respeito da necessidade das pessoas repetirem com frequência o que disseram $(p=0,030)$, sendo que os idosos do projeto Nucleação referiram uma maior necessidade de repetição que os demais. E, ao serem comparados os resultados da pesquisa das desvantagens auditivas por participação nos projetos, pode-se observar que os idosos do projeto Enriquecimento Cultural tinham mais dificuldades em ouvir quando alguém fala cochichando em relação aos idosos do PIE $(p=0,026)$.

\section{DISCUSSÃO}

Os idosos pesquisados, em sua maioria, autoavaliaram suas condições de saúde (saúde geral, visão, audição e memória) como boas ou razoáveis, sendo mais raras as auto-avaliações extremadas (excelente e ruim), o que é compatível com a literatura $6,10,11$.
É grande a referência a problemas de saúde, sendo os mais citados a hipertensão, a artrose e o Diabetes, tal qual encontramos em estudos com outras populações de idosos ${ }^{6,12-14}$. O uso de medicação regular foi identificado na maioria dos idosos, resultados que convergem com a alta prevalência de uso de medicação regular por idosos referida por outros estudos ${ }^{6,15,16}$. A maioria dos idosos entrevistados não era tabagista atual e nem havia consumido bebidas alcoólicas no mês que antecedeu a pesquisa, indicando uma baixa tendência a hábitos de vida danosos à saúde entre a população estudada.

Quando se discute as alterações da audição no envelhecimento, não há, ainda, um consenso. Alguns autores acreditam que o processo de debilidade sensorial, que se dá com o passar dos anos, seja normal e inerente a todos os seres humanos, percebido em menor ou maior grau pelos diferentes indivíduos ${ }^{17}$, enquanto outros o caracterizam como uma morbidade e recomendam sua pesquisa e intervenção adequada ${ }^{18}$.

Apesar da alta prevalência de déficit auditivo em indivíduos idosos, este é um problema pouco investigado durante as avaliações médicas neste grupo etário e, aponta-se a triagem auditiva como uma ferramenta útil na identificação da perda auditiva 
em atenção primária da saúde, por tratar-se de um problema de início insidioso, do qual os pacientes, muitas das vezes, não têm consciência ${ }^{19}$.

O teste considerado padrão na realização de avaliações auditivas em populações adultas é a audiometria, exame que necessita de profissional treinado, equipamento especializado e local com isolamento acústico (sala com tratamento acústico e cabine audiométrica). A situação ideal, para confirmação e quantificação de perdas auditivas é a submissão dos participantes das pesquisas ao exame audiométrico, no entanto a complexidade e o custo do procedimento são obstáculos a essa iniciativa.

Um complicador em estudos sobre a perda auditiva do idoso é que as medidas objetivas de detecção só explicam parcialmente o grau desse déficit, visto que as avaliações subjetivas que adultos mais velhos fazem de suas próprias deficiências, muitas vezes, não estão de acordo com os resultados encontrados em exames. Isto quer dizer que mesmo uma perda auditiva marginal (muito próxima do grau de normalidade) pode causar sensações de menor bem-estar, de incapacidade e de diminuição do funcionamento cognitivo, pois a audição é parte da integralidade do sujeito na vida cotidiana ${ }^{4}$. As avaliações audiométricas, que são consideradas meios de se quantificar a perda auditiva, não são suficientes para demonstrar a real habilidade ou dificuldade comunicativa e de desempenho psicossocial do sujeito avaliado, já que não representam as consequências emocionais, sociais e de situação percebidas em função dessa perda ${ }^{20} \mathrm{e}$, diante de tal realidade, muitos clínicos adotam os questionários estruturados, que podem ser auto-administrados, por suas características de praticidade e abrangência, com o objetivo de sanar, em parte, essa lacuna.

Considera-se que, no campo das pesquisas, os inquéritos de avaliação auditiva, além de rastrearem a perda auditiva, podem fornecer pistas sobre tendências temporais na prevalência das perdas auditivas e identificar áreas geográficas e subgrupos de populações sob risco (segundo gênero, raça, etnia) ${ }^{19}$, tornando-se útil a auto-avaliação da audição do idoso como forma de avaliar também as dimensões subjetivas da perda auditiva que não aparecem no audiograma.

Nesse estudo, apesar de se utilizar amostra reduzida, foram observadas queixas auditivas a respeito de situações do cotidiano dos idosos, que dificilmente seriam pesquisadas em avaliações de saúde de rotina e nem mesmo nas audiometrias. Para uma futura pesquisa sugerem-se amostras mais representativas e diversificadas para extrapolar os resultados para outras populações, além da possibilidade de aplicação do questionário associada a um teste objetivo, para que seja possível a avaliação da validade da audição auto-referida.
As características presentes nas queixas auditivas, encontradas nesse estudo, ou seja, a dificuldade de compreensão dos sons da fala durante o ato comunicativo, a necessidade de que as pessoas repitam o que disseram ou falem mais alto, além da necessidade de elevar o volume dos aparelhos eletrônicos, também podem ser explicadas por dificuldades de compreensão dos sons da fala, e não necessariamente por diminuição da audição. As demais queixas observadas, como a menor tolerância a sons intensos e ambientes muito ruidosos, são comuns e frequentemente citadas por outros, ao definirem e caracterizarem o processo de enveIhecimento da audição ${ }^{17}$.

Quando foram comparadas as implicações sociais da desvantagem auditiva e as implicações emocionais observou-se que o idoso tendia a sentir-se mais prejudicado em situações que envolviam terceiros, procurando isolar-se, o que poderia ser explicado pelo sentimento de frustração e incapacidade de desenvolver plenamente suas funções sociais, características já descritas na literatura. $\mathrm{O}$ déficit auditivo do idoso causa perdas de informação no contato interpessoal, dificuldades em manter-se informado pelos meios de comunicação e de utilizálos como instrumentos de lazer ${ }^{3}$. Na lista dos fatores relacionados à perda auditiva do idoso estão a redução na percepção da fala e incapacidade auditiva em diferentes situações e ambientes acústicos (reuniões familiares, igreja, teatro, ouvindo rádio ou vendo TV), alterações psicológicas tais como a depressão, frustração, raiva e medo causados pela dificuldade na comunicação; isolamento social e problemas de alerta e defesa (não ouvem buzinas, sirenes, veículos que se aproximam) ${ }^{17}$.

Pode acontecer que o idoso com déficit auditivo, consciente de que o problema na comunicação interpessoal seja gerado pela sua própria dificuldade, frequentemente submeta-se e torne-se mais silencioso e recluso, em vez de arriscar-se a sentimentos de raiva e de frustração ${ }^{4}$. Estes sentimentos podem provocar determinado afastamento do sujeito das interações sociais, possibilitando interpretação errônea de comportamento anti-social ou mesmo de início de demência.

Quanto às faixas etárias, não houve diferenças significativas para a auto-avaliação da audição, mas ao se analisar as queixas auditivas "Necessidade de que as pessoas repitam o que disseram com frequência" e a "Necessidade de colocar a TV e o rádio em volumes altos para ouvir melhor", bem como a implicação de desvantagem auditiva "A dificuldade em ouvir faz você assistir à TV ou ouvir rádio menos vezes do que gostaria?", observou-se que os idosos das faixas etárias superiores se referem a mais dificuldades que os demais, significando 
que quanto mais velhos, maiores são as dificuldades e desvantagens auditivas.

As queixas auditivas e de percepção de desvantagem ocorreram independente dos níveis sócio-econômicos, sendo verificadas em ambos os grupos, o que sugere que os serviços de saúde privados e públicos devem se preparar para absorver a demanda que, cresce, com o incremento contínuo do número de idosos.

Os resultados tornam-se significativos, principalmente, ao observar que a população de estudo foi constituída de idosos participantes de projetos que objetivavam também sua participação social, além de incluir, predominantemente, "idosos jovens" (60 a 69 anos), o que poderiam ser fatores desfavoráveis para se encontrar queixas.

\section{CONCLUSÃO}

As queixas auditivas frequentemente apontadas pelos idosos pesquisados dificilmente seriam pesquisadas em avaliações de saúde de rotina. Quanto aos impactos sociais e emocionais do handicap auditivo, estes se relacionaram predominantemente a situações de interação comunicativa, o que poderia causar dificuldades de inserção do idoso em seu meio social.

\section{AGRADECIMENTOS}

Agradecemos aos professores do NATES/UFJF pelo apoio e incentivo para a realização do nosso trabalho.

\begin{abstract}
Purpose: to investigate hearing complaints and characterize the social and emotional impacts of hearing handicap in elderly. Methods: this cross-sectional study analyzed 35 participants of the projects: Enriquecimento Cultural e Processos de Envelhecimento and Nucleação, both of Pólo Interdisciplinar na Área do Envelhecimento of the Federal University of Juiz de Fora. Two instruments were used for data collection: an inventory the social-demographic features, general health and hearing perception and a tool reffered to as The Hearing Handicap Inventory for the Elderly, HHIE (adapted by Wieselberg, 1997). We performed the descriptive analysis of the variables under study and, in order to check the association among the occurrence of hearing handicap with the others variables, bivariate analyzes were carried out, by using the $X^{2}$ test, considering $p<0.05$. Results: the results showed the prevalence of hearing complaint for at least $11(31.4 \%)$ of the people and the prevalence of hearing handicap in $10(28.6 \%)$ regarding the emotional and social issues. The main hearing handicap implications were related to the social abilities, such as conversation comprehension; TV/radio comprehension; low tolerance to intense sounds and noisy environments. Only 7 (20\%) did not have any perception of hearing problems. Conclusion: in spite of using a reduced sample, we noted hearing complaints in daily activities of elderly, which could not be investigated with routine health assessments, not even with audiometry. These results were significant, mainly because we used a specific group, that included "young elderly" (60-69-year old), facts that could decrease the number of complaints.
\end{abstract}

KEYWORDS: Self Assessment; Hearing; Aging

\section{REFERÊNCIAS}

1. Ramos LR. Fatores determinantes do envelhecimento saudável em idosos residentes em centro urbano: Projeto Epidoso, São Paulo. Cad Saúde Pública. 2003; 19(3):793-8.

2. Lima-Costa MF, Firmo JOA, Uchôa E. A estrutura da auto-avaliação da saúde entre idosos: projeto Bambuí. Rev Saúde Pública. 2004; 38(6):827-34.

3. Mansur LL, Viúde A. Aspectos fonoaudiológicos do envelhecimento. In: Papaléo Netto M. Gerontologia: a velhice e o envelhecimento em visão globalizada. São Paulo: Atheneu; 1996. p. 284-96.

4. Stuart-Hamilton I. A psicologia do envelhecimento: uma introdução. 3. ed. Porto Alegre: Artmed; 2002. 5. Sindhusake D, Mitchell P, Smith W, Golding M, Newall P, Hartley D, et al. Validation of self-reported hearing loss. The Blue Mountains Hearing Study. Int J Epidemiol. 2001; 30(6):1371-8.

6. Camarano AA. Envelhecimento da população brasileira: uma contribuição demográfica. Texto para discussão número 858. Instituto de Pesquisa 
Econômica Aplicada (IPEA). Ministério do Planejamento, Orçamento e Gestão. Rio de Janeiro; 2002. Disponível em: <http://www.ipea.gov.br/pub/ td/2002/td_0858.pdf>

7. World Health Organization. Men, ageing and health-achieving health across the life span. Genebra; Who, Noncommunicable Diseases Prevention and Health Promotion Department; 2001. (WHO/NMH/NPH/01.2)

8. Cruickshanks KJ, Wiley TL, Tweed TS, Klein BE, Klein R, Mares-Perlman JA, et al. Prevalence of hearing loss in older adults in Beaver Dam, Wisconsin. The epidemiology of hearing loss study. Am J Epidemiol. 1998; 148(9):879-86.

9. Wieselberg MB. A auto-avaliação do handicap em idosos portadores de deficiência auditiva: o uso do HHIE. 1997. [dissertação] São Paulo (SP): Pontifícia Universidade Católica; 1997.

10. Lee Y, Shinkai S. A comparison of correlates of self-rated health and functional disability of older persons in the Far East: Japan and Korea. Arch Gerontol Geriatr. 2003; 37(1):63-76.

11. Szwarcwald CL, Leal MC, Gouveia GC, Souza WV. Desigualdades socioeconômicas em saúde no Brasil: resultados da Pesquisa Mundial de Saúde, 2003. Rev Bras Matern Infant. 2005; 5(Supl1):11-22.

12. Kabir ZN, Tishelman C, Aguero-Torres $\mathrm{H}$, Chowdhury AMR, Winblad B, Höjer B. Gender and rural-urban differences in reported health status by older people in Bangladesh. Arch Gerontol Geriatr. 2003; 37(1):77-91.

13. Peixoto SV, Firmo JOA, Lima-Costa MF. Condições de saúde e tabagismo entre idosos residentes em duas comunidades brasileiras
(Projetos Bambuí e Belo Horizonte). Cad Saúde Pública. 2006; 22(9):1925-34.

14. Lima-Costa MF, Peixoto SV, Matos DL, Firmo JOA, Uchoa E. A influência de respondente substituto na percepção da saúde de idosos: um estudo baseado na Pesquisa Nacional por Amostra de Domicílios (1998-2003) e na coorte de Bambuí, Minas Gerais, Brasil. Cad Saúde Pública. 2007; 23(8):1893-902.

15. Paskulin LMG, Vianna LAC. Perfil sociodemográfico e condições de saúde autoreferidas de idosos de Porto Alegre. Rev Saúde Pública. 2007; 41(5):757-68.

16. Lima-Costa MF, Barreto SM, Giatti L. Condições de saúde, capacidade funcional, uso de serviços de saúde e gastos com medicamentos da população idosa brasileira: um estudo descritivo baseado na Pesquisa Nacional por Amostra de Domicílios. Cad Saúde Pública. 2003; 19(3):735-43.

17. Sanches EP, Suzuki HS. Fonoaudiologia em gerontologia. In: Suzuki HS, organizador. Conhecimentos essenciais para atender bem o paciente idoso. São José dos Campos: Pulso; 2003. p. 39-51.

18. Brink $P$, Stones $M$. Examination of the relationship among hearing impairment, linguistic communication, mood, and social engagement of residents in complex continuing-care facilities. Gerontol. 2007; 47(5):633-41.

19. Valete-Rosalino CM, Rozenfeld S. Triagem auditiva em idosos: comparação entre auto-relato e audiometria. Rev Bras Otorrinolaringol. 2005; 71(2):193-200.

20. Corrêa GF, Russo ICP. Autopercepção do Handicap em deficientes auditivos adultos e idosos. Rev CEFAC. 1999; 1(1):54-63.

RECEBIDO EM: 22/07/2008

ACEITO EM: 14/10/2008

Endereço para correspondência:

Lívia Maria Santiago

Rua José Libânio Rodrigues, 230/202

Juiz de Fora - MG

CEP: $36047-000$

E-mail: liviamsantiago@gmail.com 